\title{
Verrucous Carcinoma of the Foot Arising on a Muscle Flap and Skin Graft Site in a 74-year-old Filipino Female: A Case Report
}

\author{
Eileen Liesl A. Cubillan ${ }^{1}$ and Monica Felise J. Sabal ${ }^{2}$ \\ ${ }^{1}$ Department of Dermatology, College of Medicine and Philippine General Hospital, University of the Philippines Manila \\ ${ }^{2}$ Maria Reyna-Xavier University Hospital, Cagayan de Oro City
}

\begin{abstract}
Verrucous carcinoma is an uncommon low-grade well-differentiated malignant neoplasm that commonly arises on acral and mucosal sites. It is often both clinically and histologically misdiagnosed because of its slow growth and low cytologic atypia, respectively. We describe the case of a 74-year-old female with a papillomatous mass arising on a muscle flap and skin graft site on the left foot that had delay in diagnosis for more than 30 years. This case highlights verrucous carcinoma as consideration for both clinical and histologic differential diagnoses in chronic non-healing fungating tumor on the foot. Though non-aggressive in its course, the tumor can cause extensive local destruction of contiguous structures, hence, timely diagnosis and excision is paramount to prevent limb amputation.
\end{abstract}

Key Words: verrucous carcinoma, skin graft, fungating mass, muscle flap

\section{INTRODUCTION}

Verrucous carcinoma is a low-grade well-differentiated squamous cell malignancy that appears as a fungating papillomatous mass on mucosal and acral sites. It was first described by Lauren V. Ackerman in 1948 for a tumor in the oral cavity termed oral florid papillomatosis. ${ }^{1}$ Similar lesions have been reported in the soles and given the diagnosis of epithelioma cuniculatum ${ }^{2}$ which was later renamed as carcinoma cuniculatum. ${ }^{3}$ Histology of both the oral mucosa and acral neoplasms were described with markedly endophytic tongues of bulbous rete ridges and mild atypical features. Having similar histology and course, the umbrella diagnosis of "verrucous carcinoma" was coined. ${ }^{2}$

Most common causes implicated are infections such as human papillomavirus ${ }^{3}$ in plantar warts and chronic non-healing wounds such as in a diabetic patient. ${ }^{4}$ Histologically, verrucous carcinoma is characterized by low degree of atypia. It is this low cytologic atypia that makes it pathologically challenging resulting in misdiagnosis, and subsequent delay in proper management. We report a case of verrucous carcinoma arising from gastrocnemius muscle flap and skin graft of the foot following vehicular trauma.

Corresponding author: Eileen Liesl A. Cubillan, MD

Department of Dermatology

Philippine General Hospital

University of the Philippines Manila

Taft Avenue, Manila 1000, Philippines

Email: eacubillan@up.edu.ph

\section{CASE PRESENTATION}

A 74-year-old hypertensive, diabetic Filipino woman sought consult for a foul smelling mass on the left posterior ankle arising from a gastrocnemius graft site. The ulcerating, fungating painful lesion measured $7 \mathrm{~cm} \times 7 \mathrm{~cm}$ on consult. 
Around 50 years prior, the patient had a motor vehicle accident and underwent ankle fusion with gastrocnemius muscle flap and skin graft on her left foot. A slow growing papule on the posterior ankle was then noted which developed into an exophytic nodule within the span of three years, measuring approximately $2 \mathrm{~cm} \mathrm{x} 2 \mathrm{~cm}$. A year later, the tumor doubled in size which prompted medical consult and biopsy. Histologic finding then was "Pseudoepitheliomatous hyperplasia". The patient was treated with oral antibiotics (cefalexin) and pain reliever (celecoxib). No further follow-up was made by the patient and she instead underwent incision, local debridement and other local therapies by a midwife. Rapid increase in size of the mass prompted the patient to

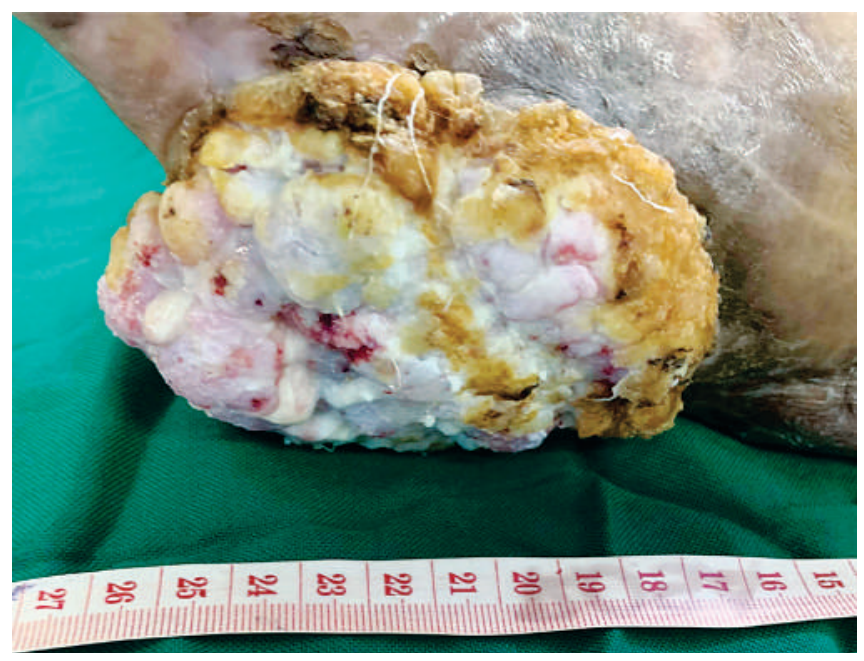

Figure 1. Verrucous, fungating tumor associated with areas of crusting and necrosis that developed from a skin graft on the plantar surface of the left posterior ankle.

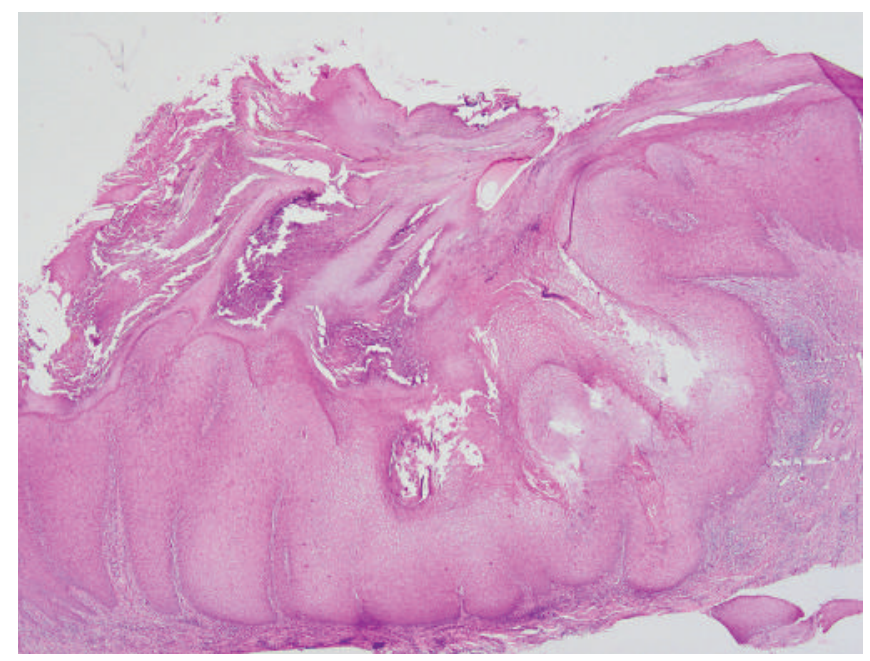

Figure 2. H\&E staining (10x) showing bulbous endophytic growth with broad pushing blunt squamous epithelial downgrowths with broad rounded bases (club-shaped). follow up and seek orthopedic consult. By that time, the mass was noted as an ulcerating, fungating tumor associated with foul smelling exudate and focal areas of necrosis arising from a hyperpigmented skin flap on the plantar surface of the left posterior ankle (Figure 1). Referral to the Section of Dermatology was made and impression of squamous cell carcinoma was given. Incisional biopsy of the tumor showed histopathologic findings of verrucous carcinoma (Figures 2 and 3).

MRI and a CT scan of the left foot showed a $7.5 \mathrm{~cm}$ lobulated soft tissue mass superficially located within the cutaneous-subcutaneous region of the posterolateral aspect of the ankle (Figures 4 and 5). There was no tumor infiltration into the deeper soft tissues of the hind foot and no evidence of cortical bone destruction and marrow invasion.

Regional lymph nodes were not enlarged. Holoabdominal ultrasound and chest radiographs were normal.

Laboratory work up showed elevated white blood cell count, fasting blood sugar and elevated glycosylated hemoglobin. She was given sultamicillin IV and started on insulin for blood glucose control.

The patient underwent a below the knee amputation of the left leg and had no postoperative complications.

\section{DISCUSSION}

Verrucous carcinoma is a slow growing neoplasm often reaching a size of more than $10 \mathrm{~cm} .{ }^{5}$ Chronic trauma can be an eliciting and aggravating factor in its development such as chewing of betel nut in Ackerman tumor ${ }^{1}$ and constant pressure at the ball of the foot in carcinoma cuniculatum. ${ }^{3}$ The ball of the foot is where pressure is most often exerted and where people often develop pain. ${ }^{3}$ In our patient, the

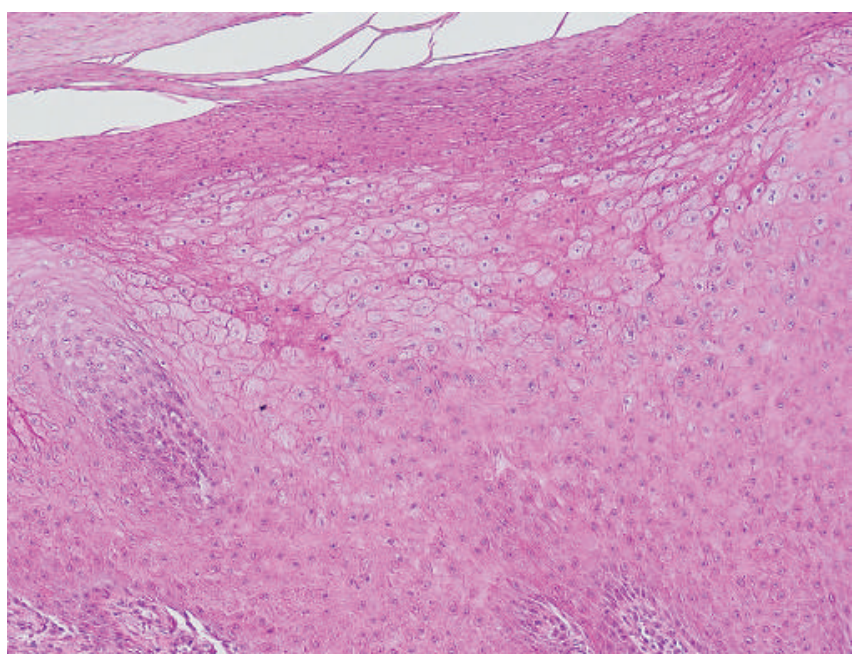

Figure 3. H\&E staining (20x) showing pale staining keratinocytes with low cytologic atypia. 

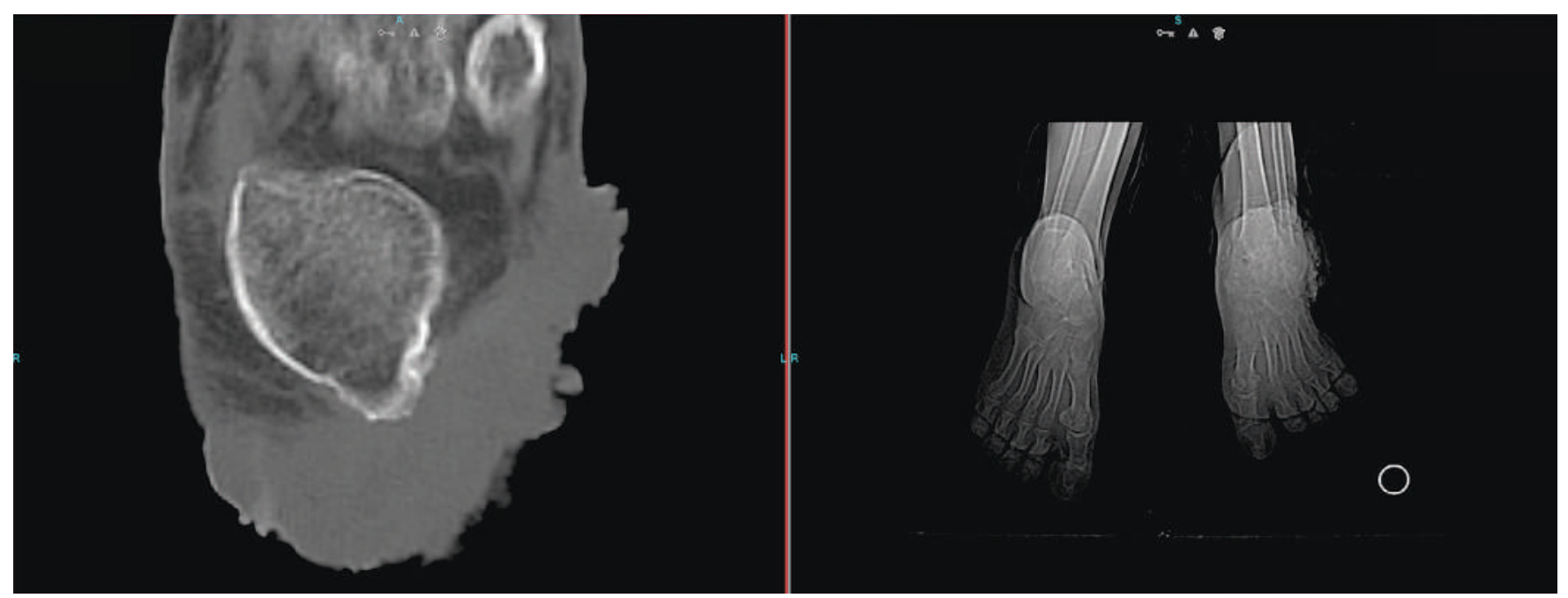

Figure 4. MRI showing lobulated mass with no evidence of cortical invasion.

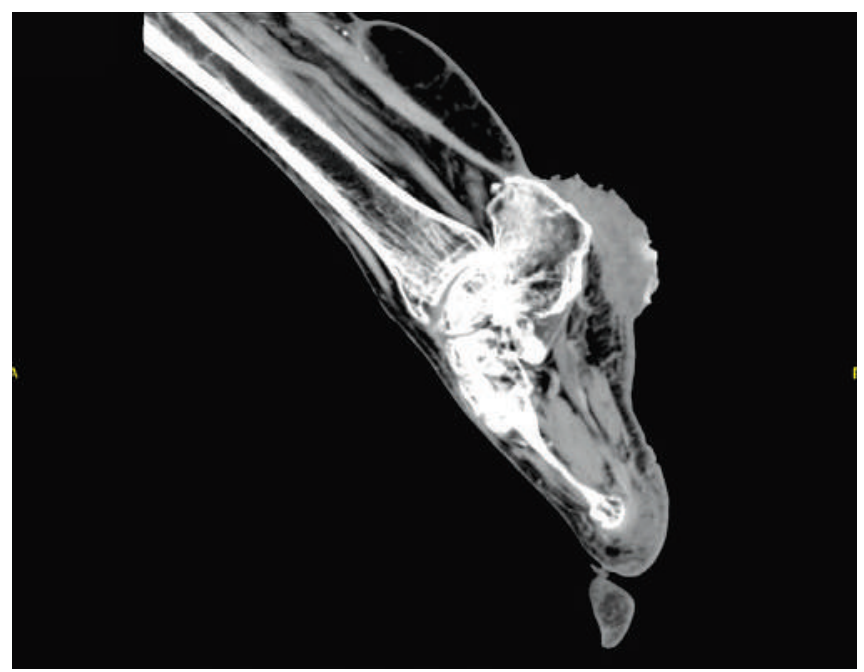

Figure 5. CT scan showed a lobulating soft tissue mass within the cutaneous and subcutaneous layer of the hindfoot extending up to the ankle.

neoplasm developed on a muscle flap and skin graft site where repeated incisions were done on several occasions. Verrucous carcinoma of the foot is uncommon in the Philippines with most of the local published reports on verrucous carcinoma of the anogenital areas. ${ }^{6-8}$ Carcinomas particularly squamous cell carcinoma have been reported on skin graft donor site with acute inflammatory process incited by the scarring hypothesized as its cause. ${ }^{9}$ However, development of verrucous carcinoma, whether on muscle flap, skin graft donor, or recipient site has not been reported in recent published literature as searched in NCBI, Pubmed, Google Scholar, and Herdin using keywords "verrucous carcinoma arising on skin graft" and "verrucous carcinoma arising on muscle flap". Therefore, to date, this is the first case report of verrucous carcinoma that developed on a muscle flap and skin graft site.
A fungating neoplasm with a gradual slow course and pseudoepitheliomatous hyperplasia on histology would have infectious process as differential diagnosis, particularly deep fungal and mycobacterial infections. However, absence of histiocytes in the dermis in a chronic nodular growth portends to non-infectious considerations. Superficial biopsies of this large mass coupled with its bland cytology on histology can also mislead to diagnosis of a benign epithelial tumor. Common misdiagnosis is verruca vulgaris with its marked papillomatosis. Though human papillomavirus infection has been implicated in some epithelioma cuniculatum $^{3}$, it is not always the cause as in our patient where koilocytic change was not evident on histological examination. Furthermore, with its massive size and its chronic non healing course, squamous cell carcinoma is a consideration. However, the clinical extent of the neoplasm seemingly incongruous with the histologic absence of frank atypia in the form of nuclear hyperchromasia, mitotic figures, horn pearls and invasive dermal epithelial islands, make this more aggressive form of carcinoma much less likely.

Verrucous carcinoma is characterized histologically by silhouette of marked papillomatosis, peripheral buttressing, and shouldering. ${ }^{5}$ Similar silhouette and the glassy cytoplasm of the keratinocytic proliferation are features similarly seen in keratoacanthoma. However, the clinical picture and the 30-year course of our patient's tumor is incompatible with that of the rapid growth and eventual spontaneous involution of a keratoacanthoma. Hence, clinicopathologic correlation is paramount and its emphasis much displayed in this case.

Finally, a form of skin graft rejection may also be considered as possible factor in the development of verrucous carcinoma. Chronic irritation and inflammation most probably contributed to its malignant transformation. ${ }^{6}$ In the lower limbs such as the foot, skin grafts have been shown to have higher failure and complication rates compared to other areas of the body. ${ }^{10}$ Skin grafts were noted to fail 
usually within 6 weeks. ${ }^{11}$ Our patient noted development of a $2 \times 2 \mathrm{~cm}$ nodule within 3 years of the graft. The factors that cited increased risk of rejection were increased BMI, peripheral vascular disease, and immunosuppressant medication. ${ }^{11}$ Our patient was not obese and did not exhibit peripheral vascular disease. However, she was found to have elevated glycosylated hemoglobin and hence may have been an undiagnosed and untreated diabetic which possibly contributed to an increased risk of graft rejection.

The prognosis of verrucous carcinoma is often good as it does not have metastatic potential ${ }^{2}$ though it may invade adjacent tissue and bone structures. ${ }^{12}$ MRI and x-rays of our patient showed no evidence of cortical bone destruction and marrow invasion.

This case highlights the importance of early diagnosis and management of verrucous carcinoma as wide excision is curative with distant metastasis uncommon in its course. Superficial biopsies of a markedly fungating neoplasm often leads to misdiagnosis hence inclusion of the deep dermis and subcutaneous fat is essential as histologic diagnosis depends on the architecture and silhouette of the neoplasm. Lastly, high index of suspicion, both clinically and histologically is important to enable correct diagnosis and prompt management to avoid further local invasion and eventual amputation which could have been prevented in our patient.

\section{Statement of Authorship}

All authors participated in data collection and analysis, and approved the final version submitted.

\section{Author Disclosure}

All authors declared no conflicts of interest.

\section{Funding Source}

This paper was self-funded.

\section{REFERENCES}

1. Ackerman LV. Verrucous carcinoma of the oral cavity. Surgery. 1948; 23(4):670-8.

2. Brownstein MH, Shapiro L. Verrucous carcinoma of skin: epithelioma cuniculatum plantare. Cancer. 1976; 38(4):1710-6.

3. Coldiron BM, Brown FC, Freeman RG. Epithelioma cuniculatum (Carcinoma cuniculatum) of the thumb: a case report and literature review. J Dermatol Surg Oncol. 1986; 12(11):1150-5.

4. Sarma D, Wang J, Bewtra C, Lee L. Verrucous carcinoma arising in a chronic non-healing ulcer of the foot of a diabetic patient. Internet J Dermatol. 2006;5(1):4-7.

5. Aird I, Johnson HD, Lennox B, Stansfeld AG. Epithelioma cuniculatum, a variety of squamous carcinoma peculiar to the foot. Br J Surg. 1954; 42(173):245-50.

6. Ong N, Gabriel T. Verrucous carcinoma of the penis not associated with HIV. J Philipp Dermatol Soc. 2008; 17(2):92-4.

7. Sjambali S, Gabriel T. Carcinoma cuniculatum, an anogenital type of verrucous carcinoma: a case report. J Philipp Dermatol Soc. 2012; 21(1):17-20.

8. Vasquez OG, Cubacub EA. Verrucous carcinoma of the larynx. DLSMC Scientific Journal 1983. Abstract retrieved from Herdin.ph database.

9. Herard C, Arnaud D, Goga D, Rousseau P, Potier B. Rapid onset of squamous cell carcinoma in a thin skin graft donor site. Ann Dermatol Venereol. 2016; 143(6-7):457-61.

10. Henderson NJ, Fancourt M, Gilkison W, Kyle S, Mosquera D. Skin grafts: a rural general surgical perspective. ANZ J Surg. 2009; 79(5):362-6.

11. Reddy S, El-Haddawi F, Fancourt M, Farrant G, Gilkison $\mathrm{W}$, Henderson N, et al. The incidence and risk factors for lower limb skin graft failure. Dermatol Res Pract. 2014;2014(582080).

12. Reingold IM, Smith BR, Graham JH. Epithelioma cuniculatum pedis, a variant of squamous cell carcinoma. Am J Clin Pathol. 1978; 69(5):561-5. 